\title{
Génération d'harmoniques élevées limitée par l'absorption : le microjoule atteint à $53 \mathrm{~nm}$
}

\author{
J.-F. Hergott, M. Kovacev, H. Merdji, C. Hubert, Y. Mairesse, E. Jean, P. Breger, \\ P. Agostini, P. Salières et B. Carré
}

Service des Photons, Atomes et Molécules, CEA-Saclay, 91191 Gif-sur-Yvette, France

\begin{abstract}
Résumé : Nous étudions les harmoniques d'ordre élevé générées par un laser de forte énergie focalisé avec une lentille de grande focale. Nous identifions différents processus qui peuvent limiter l'efficacité de génération: le désaccord de phase, l'absorption, et la défocalisation du laser. Dans les conditions optimales, nous obtenons une grande efficacité de conversion permettant une augmentation d'au moins un ordre de grandeur de l'énergie harmonique par rapport aux valeur rapportées jusqu'à présent. Dans le xénon, nous atteignons le microjoule, ce qui ouvre la voie à de nouvelles applications de ce rayonnement cohérent ultrabref dans l'UVX.
\end{abstract}

\section{INTRODUCTION}

La génération des harmoniques d'ordre élevé d'impulsions laser intenses dans un gaz a ouvert depuis peu de nouvelles perspectives pour sonder la matière dans l'UVX à une échelle de temps encore jamais atteinte. La durée ultracourte des impulsions harmoniques est utilisée dans des expériences pompe-sonde, en spectroscopie atomique et moléculaire comme en physique du solide. Combinée à la haute cohérence intrinsèque du faisceau, elle a permis le diagnostic ultra rapide par interférométrie UVX de plasmas produits par lasers. Cependant, l'énergie du faisceau harmonique reste relativement faible, et de nouvelles applications seraient possibles si le nombre de photons harmoniques était augmenté : l'holographie UVX ultra rapide, le diagnostic de plasmas denses brillants, ou encore l'étude de processus non linéaires dans I'UVX, limitée jusqu'à présent à des ordres harmoniques faibles. Récemment, plusieurs études ont démontré de grandes efficacités de conversion en utilisant des impulsions laser ultrabrèves focalisées dans des fibres creuses [1,2] ou des cellules[3,4]. Cependant, ces efficacités étaient obtenues à très faible énergie laser (moins d' $1 \mathrm{~mJ}$ dans la plupart des cas), ce qui imposait une focalisation assez forte pour atteindre les éclairements de saturation des gaz rares de génération. Il en résultait une énergie harmonique faible, de l'ordre du nanojoule. Le fait que des énergies bien plus élevées sont désormais disponibles sur les systèmes laser ultracourts soulève un certain nombre de questions : En utilisant des énergies laser plus importantes et une focalisation plus faible, est-il possible d'atteindre des efficacités similaires, et ainsi de générer des impulsions harmoniques microjoules? En particulier, quelles modifications ces conditions de génération inhabituelles apporteront-elles à l'accord de phase?

\section{RESULTATS ET DISCUSSION}

Nos expériences ont été réalisées sur le dispositif laser LUCA avec un système Titane : Saphir délivrant des impulsions de $60 \mathrm{fs}$ à $800 \mathrm{~nm}$, avec une énergie pouvant atteindre $100 \mathrm{~mJ}$ à $20 \mathrm{~Hz}$. Dans notre expérience, nous focalisons jusqu'à $27 \mathrm{~mJ}$ dans un jet de gaz pulsé avec une lentille de focale $2 \mathrm{~m}$ ou $5 \mathrm{~m}$. Une buse présentant une fente de dimension $300 \mu \mathrm{m} * 3 \mathrm{~mm}$ produit un jet d'une pression de 10 à 100 Torr. En tournant le jet par rapport à l'axe du laser, on peut changer la longueur du milieu générateur tout en gardant la même densité crête. Les harmoniques produites dans le jet sont analysées par un spectromètre sans fente d'entrée, et détectées par une photodiode UVX calibrée protégée de la lumière $\mathbb{R}$ par deux filtres d'aluminium de $100 \mathrm{~nm}$. La réponse absolue du spectromètre et la transmission des filtres ont été mesurées en utilisant la source harmonique préalablement monochromatisée (avec un autre spectromètre).

L'ouverture totale du faisceau laser étant de $40 \mathrm{~mm}$, l'éclairement au foyer de la lentille de $2 \mathrm{~m}$ atteignait $4 * 10^{15} \mathrm{~W} / \mathrm{cm}^{2}$ (le faisceau laser est deux fois limité par la diffraction). Cette valeur esi bien 
supérieure à l'éclairement de saturation des gaz rares, ce qui limite l'efficacité de génération notamment à cause des effets d'ionisation: déplétion du milieu générateur, importante dispersion due aux électrons libres menant à un mauvais accord de phase. La première étape dans l'optimisation du signal harmonique était donc de contrôler l'ouverture du faisceau laser et la position jet/foyer. La fermeture d'un diaphragme placé avant la lentille implique à la fois une décroissance de l'énergie laser et un agrandissement du point focal, et par conséquent une décroissance rapide de l'éclairement focal. Dans tous les gaz, nous observons une forte augmentation du signal harmonique (et une augmentation de l'efficacité de conversion encore plus forte), suivie d'une décroissance pour des ouvertures trop petites. Dans le xénon, la taille optimale était de $13 \mathrm{~mm}$, ce qui correspond à une énergie laser de $4.3 \mathrm{~mJ}$ et un éclairement de $8 * 10^{13} \mathrm{~W} / \mathrm{cm}^{2}$, dans l'argon $15 \mathrm{~mm}\left(5 \mathrm{~mJ}\right.$ et $\left.1.4^{*} 10^{14} \mathrm{~W} / \mathrm{cm}^{2}\right)$ et dans le néon $19 \mathrm{~mm}\left(8.5 \mathrm{~mJ}\right.$ et $\left.3.7 * 10^{14} \mathrm{~W} / \mathrm{cm}^{2}\right)$. Ces valeurs d'ouverture optimisent la répartition spatiale d'éclairement laser dans chacun des gaz: il faut un éclairement suffisamment élevé (légèrement en dessous de l'éclairement de saturation) sur un grand volume émetteur (diamètre de la tâche focale entre 350 et $530 \mu \mathrm{m}$ ). En général, la position optimale du jet était quelques centimètres avant le foyer.
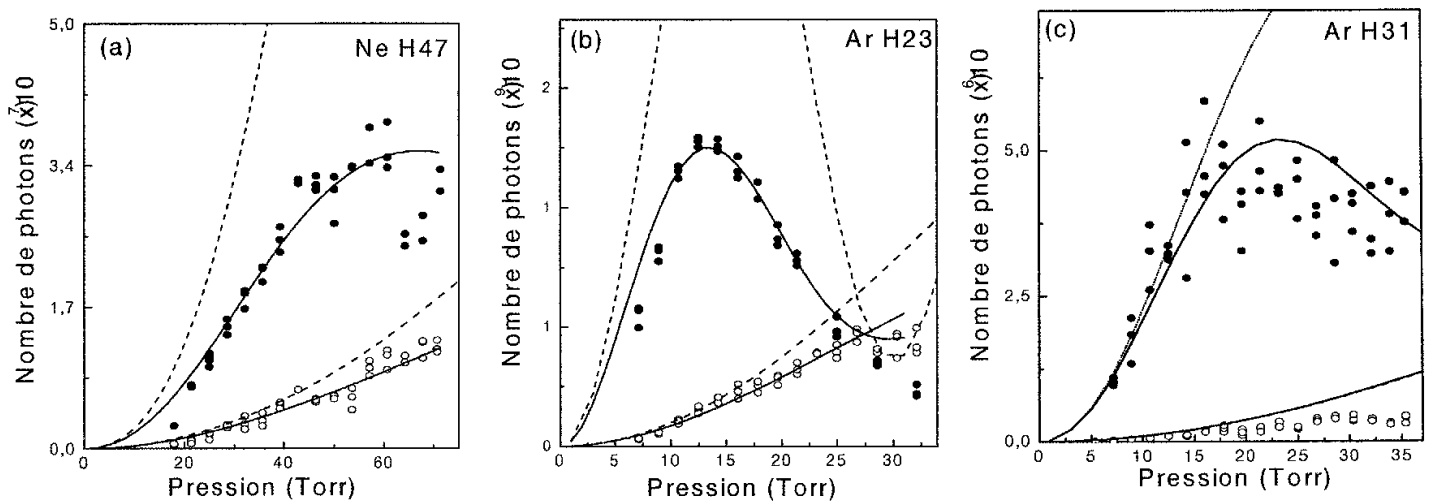

Figure 1. Signal harmonique en fonction de la pression pour H47 dans le néon (a), H23 (b) et H31 (c) dans l'argon pour un milieu de $0.5 \mathrm{~mm}$ (cercles blancs) ou $2.5 \mathrm{~mm}$ (cercles noirs). Les courbes représentent les simulations avec absorption (trait plein), sans absorption (en tirets pour (a) et (b)), et sans la défocalisation du laser (pointillés pour (c)).

Nous avons ensuite étudié les influences combinées de la pression et de la longueur du milieu à l'ouverture laser optimale. La Figure 1 représente la dépendance en pression des différentes harmoniques pour deux longueurs de milieu : $0.5 \mathrm{~mm}$ et $2.5 \mathrm{~mm}$. Le signal harmonique dans le milieu court est faible et croît lentement avec la pression. En revanche, dans le milieu long, il augmente rapidement et sature à haute pression (H47 dans le néon et $\mathrm{H} 31$ dans l'argon), ou montre une décroissance marquée après un optimum à 13 Torr dans le cas de H23 dans l'argon.

Ces variations du signal harmonique peuvent être interprétées dans un modèle $1 \mathrm{D}$ au travers des valeurs relatives des différentes longueurs caractéristiques. Le champ harmonique se construit efficacement sur la longueur de cohérence définie par $L \operatorname{coh}=\pi / a b s(\Delta k)$ où $\Delta k=k q-q k 1$ est la différence des vecteurs d'onde de l'harmonique et de la polarisation. Ce facteur d'accord de phase est déterminé par différents termes de dispersion. La dispersion atomique a un signe opposé à celui des dispersions électronique et géométrique (phase de Gouy), et peut donc les compenser à une certaine pression. En régime de champ fort, la phase «intrinsèque » du dipôle harmonique introduit un terme supplémentaire de dispersion proportionnel au gradient de l'éclairement laser I [5]. La seconde longueur caractéristique est la longueur d'absorption, définie par Labs $=1 / \sigma \rho$, où $\sigma$ est la section efficace de photoionisation, et $\rho$ la densité du gaz. Enfin, la longueur de référence est la longueur du milieu Lmed. Constant et al. [2] ont trouvé une règle empirique pour optimiser l'émission harmonique sous la forme de deux conditions : Lmed $>3$ Labs et Lcoh $>5$ Labs. Dans le milieu court, la première condition n'est pas vérifiée et l'émission est limitée par la longueur du milieu. En revanche, dans le milieu long, il y a une gamme de pressions où les deux conditions peuvent être vérifiées simultanément. L'émission est alors limitée par l'absorption, ce 
qui explique la saturation du signal harmonique sur les Figures 1a) et 1b). Cependant, la saturation observée pour H31 générée dans 1'argon (Figure 1c)) a une toute autre origine. En effet, ce modèle 1D ne prend pas en compte les effets radiaux qui interviennent lors de la propagation des champs et qui peuvent affecter le signal harmonique de manière significative, comme la défocalisation du faisceau laser par un fort gradient de densité électronique, ou un accord de phase hors axe du faisceau harmonique. L'ionisation et la phase intrinsèque du dipôle dépendent fortement de l'éclairement, et donc de la position dans le volume de focalisation et du temps via l'enveloppe temporelle du laser.

Des simulations 3D sont donc nécessaires pour interpréter sans ambiguïté la saturation du signal harmonique sur la Figure 1. Nous avons utilisé les dipôles harmoniques calculés dans l'approximation de champ fort de Lewenstein et al. [6], les taux d'ionisation tunnel de Ammosov-Delone-Krainov [7], et un code de propagation 3D [8] prenant en compte tous les effets d'ionisation (défocalisation, déplétion, désaccord de phase). Les résultats sont représentés en traits continus sur la Figure 1. L'accord avec les courbes expérimentales est excellent dans le néon et l'argon. L'amplitude des simulations a été multipliée par un même facteur correctif pour les milieux long et court afin de les superposer aux courbes expérimentales. Ceci constitue un bon test de la validité des simulations. Si l'on supprime les termes d'absorption dans le code, on obtient les courbes représentées en tirets sur les Figures 1a) et 1b). Dans le milieu court, l'influence est assez faible, ce qui indique que le facteur limitant est la longueur du milieu. En revanche, dans le milieu long, on observe un écart important des courbes à la pression optimale, ce qui indique que le régime d'émission limitée par l'absorption est atteint. Dans le cas de H23 dans l'argon, le désaccord de phase prend de l'importance à haute pression, ce qui explique la décroissance du signal harmonique due à une interférence destructive dans le milieu. A notre connaissance, c'est la première fois que la compétition entre l'absorption et l'accord de phase est si clairement observée et simulée.
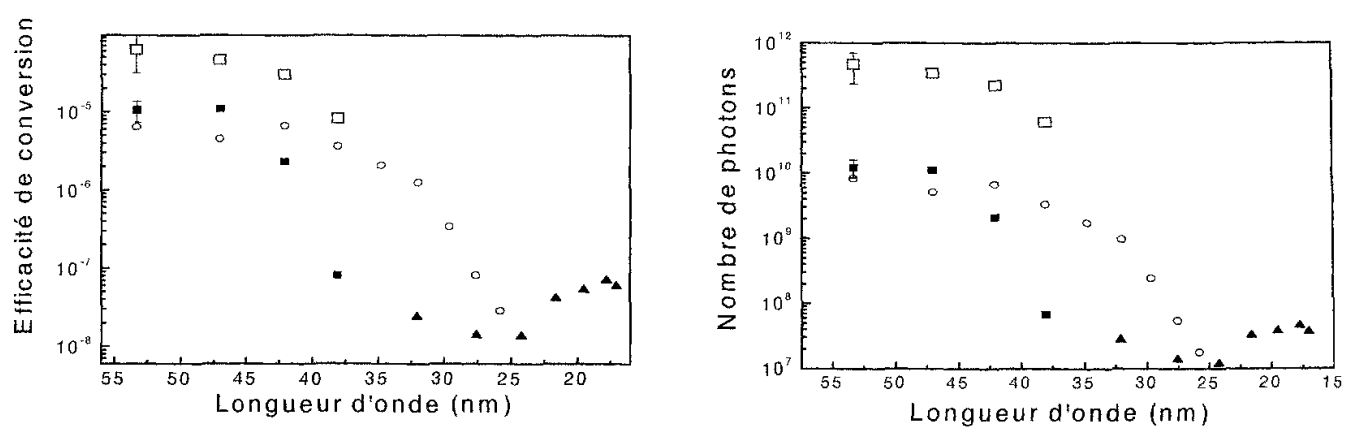

Figure 2. Nombre absolu de photons par impulsion (a) et efficacité de conversion correspondante (b) obtenus dans le xénon (carrés noirs), l'argon (cercles) et le néon (triangles noirs) avec une focale de $2 \mathrm{~m}$. Les carrés blancs montrent les résultats obtenus avec une focale de $5 \mathrm{~m}$.

Bien que la forme des courbes soit similaire, le cas de H31 générée dans l'argon est différent des précédents. Le fait d'enlever l'absorption modifie peu la dépendance en pression dans le milieu long. En effet, la section efficace d'absorption à cette longueur d'onde (proche du minimum de Cooper) est très faible, et la longueur d'absorption est de $1 \mathrm{~cm}$ à 25 Torr. En fait, la saturation du signal résulte de la défocalisation du laser et de la décroissance d'éclairement qui en découle, puisque lorsque ce phénomène n'est pas pris en compte (pointillés sur la figure 1c), le signal augmente rapidement dans le milieu long et ne présente pas de saturation dans cette gamme de pression.

Le nombre de photons harmoniques générés dans les conditions optimisées, et les efficacités de conversion correspondantes, sont représentés en Figure 2. L'efficacité atteint $10^{-5}$ pour les harmoniques du plateau du xénon, $7 * 10^{-6}$ dans l'argon, et près de $10^{-7}$ dans le néon. Le nombre de photons maximum $\left(10^{10}\right)$ est obtenu pour H15 générée dans le xénon. Dans ce cas, les dépendances en pression dans les milieux court et long sont similaires, avec une saturation aux alentours de 80 Torr, ce qui indique que l'émission est limitée par l'absorption même dans le milieu court.

Nous avons ensuite utilisé une lentille de focale $5 \mathrm{~m}$ pour augmenter la taille de la tâche focale en essayant d'utiliser toute l'énergie laser disponible. Le laser est focalisé sur la section large $(2.5 \mathrm{~mm})$ du jet de xénon. L'ouverture optimale du faisceau laser $(33 \mathrm{~mm})$ correspond à un paramètre confocal proche de 
celui utilisé avec la focale de $2 \mathrm{~m}$, mais à une énergie bien plus élevée $(25 \mathrm{~mJ})$. La position optimale du jet est alors obtenue en focalisant $11 \mathrm{~cm}$ après le milieu, comme le montre la Figure 3.

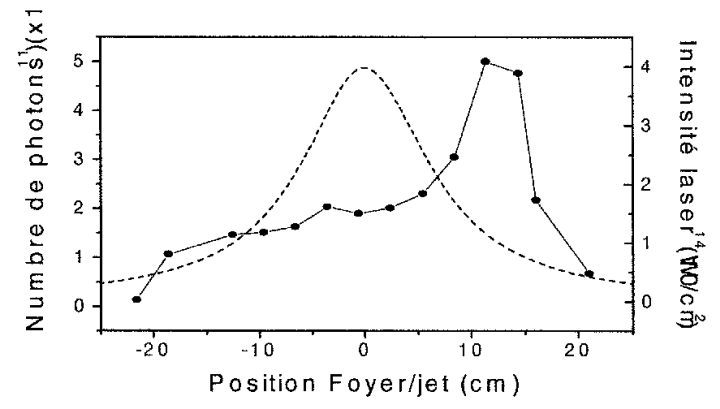

Figure 3. Nombre de photons de l'harmonique 15 générée dans le xénon ( 80 torr) avec une énergie laser de $25 \mathrm{~mJ}$ en fonction de la position relative jet/foyer. L'éclairement laser dans le vide est représenté en tirets.

A cette position, le diamètre de la tâche laser est d'environ $1 \mathrm{~mm}$, ce qui correspond à un éclairement de $1.2 * 10^{14} \mathrm{~W} / \mathrm{cm}^{2}$, légèrement au dessus de la saturation du xénon. $5 \pm 2.5 * 10^{11}$ photons sont générés (carré blanc en Figure 3), soit une énergie du faisceau harmonique de $1.9 \pm 0.9 \mu \mathrm{J}$ par impulsion. Cette valeur est supérieure de plus d'un ordre de grandeur à toutes celles précédemment rapportées. De plus, tandis que l'énergie harmonique est multipliée par 40, l'énergie de pompe ne l'est que par 6 (et l'éclairement par 1.7). L'efficacité de conversion est par conséquent augmentée d'un facteur $6 \pm 3$. Le simple effet d'échelle dû à la modification de l'énergie laser et de la taille de la tâche focale ne peut pas expliquer une telle augmentation. Elle est en fait due à l'augmentation de l'éclairement auquel une émission limitée par l'absorption est obtenue [9]. Ceci est possible grâce à la défocalisation du laser par le gradient de densité électronique : lorsque le laser est focalisé avant le milieu, sa divergence naturelle est augmentée, diminuant la longueur de génération. Lorsqu'il est focalisé après le jet, sa convergence est réduite, et les répartitions d'éclairement et de phase sont uniformes grâce à l'effet de guidage du faisceau laser.

\section{CONCLUSION}

Nous avons montré que nous avons atteint une émission harmonique limitée par l'absorption. Les simulations numériques, qui présentent un excellent accord avec les mesures expérimentales ont permis de différencier les cas où l'émission harmonique est limitée par l'absorption, des cas où elle l'est par l'accord de phase dans le milieu générateur. Nous avons également montré que des efficacités de conversion élevées peuvent être obtenues avec une grande énergie du laser de pompe en utilisant des lentilles de grandes focales. Il en résulte une augmentation d'au moins un ordre de grandeur des énergies harmoniques par rapport à celles rapportées jusqu'à présent. Dans le xénon, l'énergie mesurée de la $15^{\text {ème }}$ harmonique atteint le microjoule. Ceci ouvre la voie à de nouvelles applications du rayonnement harmonique, d'autant plus que ces résultats sont obtenus avec un équipement standard (laser $60 \mathrm{fs} 30 \mathrm{~mJ}$, jet pulsé) disponible dans de nombreux laboratoires.

\section{Références}

[1] Rundquist A. et al., Science 280 (1998) 1412.

[2] Constant E. et al., Phys. Rev. Lett. 82 (1999) 1668.

[3] Schnürer M. et al., Phys. Rev. Lett. 83 (1999) 722.

[4] Tamaki Y. et al., Phys. Rev. Lett. 82 (1999) 1422.

[5] Salières P. et al., Science 292 (2001) 902.

[6] Lewenstein M. et al., Phys. Rev. A 49 (1994) 2117.

[7] Ammosov M. V. et al., Sov. Phys. JETP 64 (1986) 1191.

[8] LHuillier A.et al., Phys. Rev. A 46 (1992) 2778.

[9] Balcou Ph. et al., in Multiphoton Processes, AIP Conference Proceedings 525, 319 (2000). 